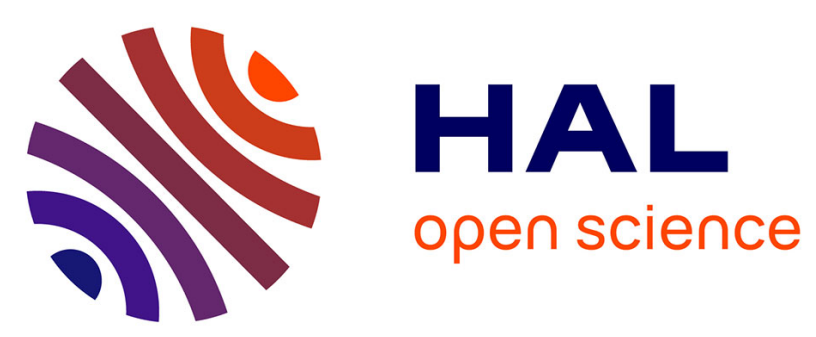

\title{
Non-destructive phase and intensity distributed measurements of the nonlinear stage of modulation instability in optical fibers
}

Arnaud Mussot, Stefano Trillo, Pascal Szriftgiser, Alexandre Kudlinski, Matteo Conforti, François Copie, Corentin Naveau

\section{To cite this version:}

Arnaud Mussot, Stefano Trillo, Pascal Szriftgiser, Alexandre Kudlinski, Matteo Conforti, et al.. Non-destructive phase and intensity distributed measurements of the nonlinear stage of modulation instability in optical fibers. Photonics West, Jan 2018, San Francisco, United States. pp.14, 10.1117/12.2295714. hal-02393427

\section{HAL Id: hal-02393427 https://hal.science/hal-02393427}

Submitted on 6 Dec 2019

HAL is a multi-disciplinary open access archive for the deposit and dissemination of scientific research documents, whether they are published or not. The documents may come from teaching and research institutions in France or abroad, or from public or private research centers.
L'archive ouverte pluridisciplinaire HAL, est destinée au dépôt et à la diffusion de documents scientifiques de niveau recherche, publiés ou non, émanant des établissements d'enseignement et de recherche français ou étrangers, des laboratoires publics ou privés. 


\title{
Non-destructive phase and intensity distributed measurements of the nonlinear stage of modulation instability in optical fibers
}

\begin{abstract}
Arnaud Mussot, Corentin Naveau, Pascal Szriftgiser, François Copie, Alexandre Kudlinski, et al.
\end{abstract}

Arnaud Mussot, Corentin Naveau, Pascal Szriftgiser, François Copie, Alexandre Kudlinski, Matteo Conforti, Stefano Trillo, "Non-destructive phase and intensity distributed measurements of the nonlinear stage of modulation instability in optical fibers," Proc. SPIE 10517, Real-time Measurements, Rogue Phenomena, and Single-Shot Applications III, 105170F (15 February 2018); doi: $10.1117 / 12.2295714$

SPIE. Event: SPIE LASE, 2018, San Francisco, California, United States 


\title{
Non-destructive phase and intensity distributed measurements of the nonlinear stage of modulation instability in optical fibers
}

\author{
Arnaud Mussot ${ }^{1}$, Corentin Naveau ${ }^{1}$, Pascal Szriftgiser ${ }^{1}$, François Copie ${ }^{1}$, Alexandre Kudlinski ${ }^{1}$, \\ Matteo Conforti ${ }^{1}$ and Stefano Trillo ${ }^{2}$ \\ 1. Univ. Lille, CNRS, UMR 8523-PhLAM - Physique des Lasers Atomes et Molécules, F-59000 \\ Lille, France \\ 2. Department of Engineering, University of Ferrara, Via Saragat 1, 44122 Ferrara, Italy \\ Authore-mail address: arnaud.mussot@univ-lille1.fr
}

\begin{abstract}
We report a novel experimental setup to perform distributed characterization in intensity and phase of the nonlinear stage of modulation instability by means of a non-invasive experimental setup : a heterodyne time domain reflectometer.
\end{abstract}

Keywords: Nonlinear fiber optics, four wave mixing, modulation instability, soliton

\section{INTRODUCTION}

Modulation instability (MI) is a key nonlinear process in physical systems where the dynamic is ruled by the focusing nonlinear Schrödinger equation (NLSE) [1]. It consists in the exponential growth of a small modulation at the expense of a strong pump wave, leading to the amplification of subsequent harmonics of the sidebands due to four-wave mixing (FWM). By further propagating in the fiber, this energy exchange between modes is reversible, the system being able to return back to its initial state. By analogy, it is usually referred to as Fermi-Pasta-Ulam (FPU) recurrence process [2,3] that is strongly connected with many complex nonlinear effects of high interest such as rogue wave generation or frequency comb generation for instance. The first experimental observation of FPU in optical fibers has been achieved in 2000's with an indirect recording of the longitudinal evolution of the pump and signal intensities. To our knowledge, the longitudinal evolution of the relative phase of these waves that is at the heart of the reversibility has never been observed. In this work, we report a full longitudinal characterization in phase and intensity of the FPU recurrence process by means of a newly designed experimental setup based on an original heterodyne detection technique.

We consider the NLSE :

$$
i \frac{\partial E}{\partial Z}-\frac{\beta_{2}}{2} \frac{\partial^{2} E}{\partial T^{2}}+\gamma|E|^{2} E=0
$$

where $E(Z, T)$ is the electric field envelope, $Z$ and $T$ the distance along the fiber and the retarded time, respectively. $\beta_{2}$ is the group velocity dispersion which is anomalous in our case and $\gamma$ the nonlinear coefficient. By means of a linear stability analysis, one can find that MI occurs when the modulation frequency $\Omega$ is such that $\Omega<\Omega_{c}$ with $\Omega_{c}=2 \sqrt{\gamma P_{p} /\left|\beta_{2}\right|}, P_{p}$ being the pump power. The MI gain peak is located at $\Omega_{\max }=\Omega_{c} / \sqrt{2}$. Fig. 1 . shows a numerical integration of the NLSE in the case of an initial pure frequency modulation (that is to say with a relative phase between the pump and the sideband of $\Delta \phi=\pi / 2$ ) with a modulation frequency close to $\Omega_{\text {max }}$ and an initial sideband to pump ratio of $-20 \mathrm{~dB}(\eta=0.01)$.

Real-time Measurements, Rogue Phenomena, and Single-Shot Applications III, edited by Bahram Jalali, Daniel R. Solli, Günter Steinmeyer, Proc. of SPIE Vol. 10517, 105170F

(C) 2018 SPIE $\cdot$ CCC code: 0277-786X/18/\$18 · doi: 10.1117/12.2295714 


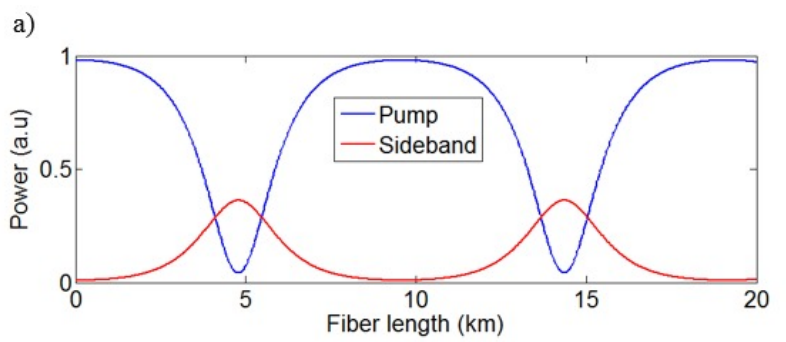

b)

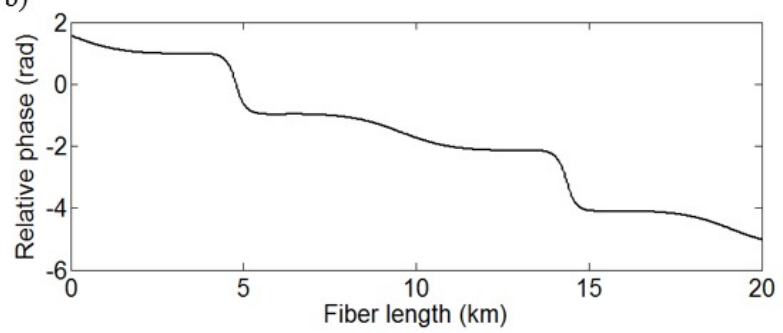

c)

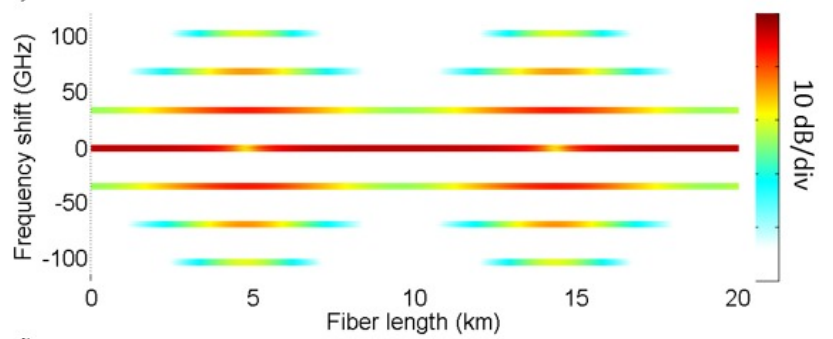

d)

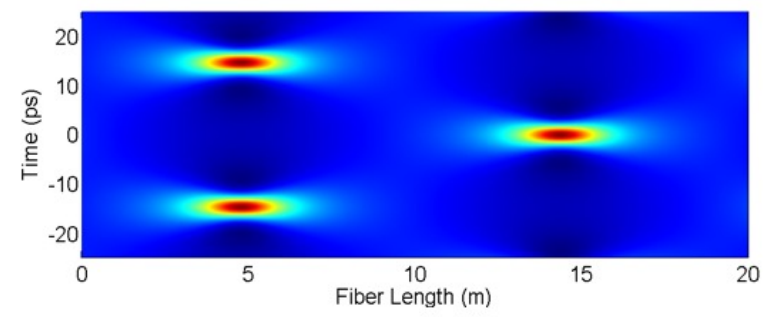

Fig. 1. Longitudinal evolution of (a) the normalized pump and sideband powers and (b) the pump-sideband relative phase. (c) and (d) shows the spectral and the temporal evolution of the intensity along the fibre, respectively. Parameters: fibre length $\mathrm{L}=20 \mathrm{~km}$, $\beta_{2}=-19 \times 10^{-27} \mathrm{~s}^{2} / \mathrm{m}, \gamma=1.3 \times 10^{-3} / \mathrm{W} / \mathrm{m}, P_{p}=450 \mathrm{~mW}$, sideband to pump ratio $\eta=0.01$, sideband detuning 35 GHz. Powers are normalized to their maximums values.

The evolution of the pump powers (Fig. 1.a.) clearly exhibits FPU recurrences. A first return to the initial state is observed around $10 \mathrm{~km}$ and another one at $18 \mathrm{~km}$.

The first experimental observation of the FPU stage of modulation instability in fiber optics was reported in 2001 [3]. Various studies have since reported distributed measurements of the FPU stage of MI but only in intensity [4]. To date evolution of the phase was not taken into account although it can drastically affect the dynamics of the system [5]. Moreover, they were limited to the observation of only one recurrence, due to the intrinsic losses of fibres which kill the FPU process [4]. Here we propose a non-invasive method to perform distributed measurements of both intensity and phase of the MI process with compensation of the losses: a heterodyne optical time domain reflectometer (HOTDR). OTDRs use the Rayleigh backscattered signal which is directly proportional to the propagating signal. A simplified sketch of the experimental setup is presented in Fig. 2:

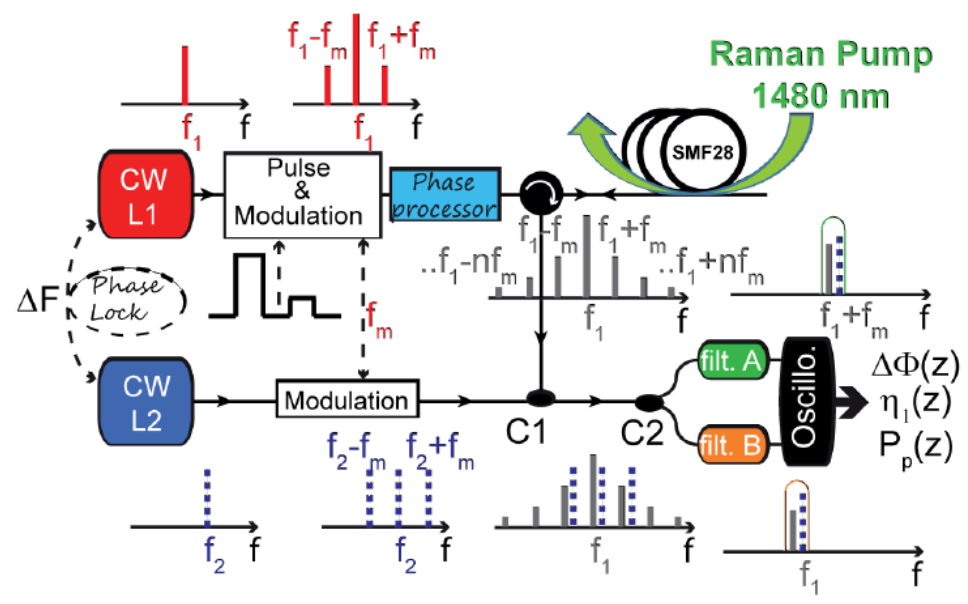

Fig. 2. Simplified sketch of the experimental setup. $f_{1,2}$ are the frequencies of the pump laser and the local oscillator laser, respectively, with_ $\Delta F=f_{1}-f_{2}=800 \mathrm{MHz}$ Here $f_{m}=35 \mathrm{GHz}$ is the input modulation frequency. MI evolution is studied in a 7.7-kmlong single mode fibre (SMF28). The backscattered signal from the SMF28 goes through a circulator and is analysed via heterodyning (beating with the local oscillator) and then filtered (filt. A and B) to isolate the power and phase evolutions of the pump and the first order sideband in the MI spectral comb. 
Intensity modulation of a CW laser (pump laser) centred at $1555 \mathrm{~nm}$ is used to generate 50-ns-long pulses. This duration is small enough to prevent any detrimental Brillouin scattering. The pump is then phase modulated in order to generate two sidebands symmetrically located around the pump and whose relative intensity and phase (compared to the pump) are controlled by an all fiber phase controller (waveshaper). After amplification, the modulated wave is launched into a 7.7-km-long SMF28 fibre. To compensate for the losses, we use a counter-propagating Raman pump centred at $1480 \mathrm{~nm}$. The Rayleigh backscattered signal is mixed with a detuned local oscillator to perform heterodyne detection. This local oscillator consists in a CW laser which is detuned by $800 \mathrm{MHz}$ and phase locked with respect to the pump. An intensity modulator driven by the same radio frequency source than the previous phase modulator is used to provide local oscillators for the previous sidebands or their harmonics.

Experimentally, the parameters we used are the same than in the simulation of Fig. 1. except for a shorter fibre length (in order to perform a good compensation of the losses) and an increased initial sideband to pump ratio (-8.5 dB) in order to scale down the recurrences. The experimental results are presented in Fig. 3. Fig. 3.a. shows that we were able to observe almost two recurrences in intensity. Moreover we managed to obtain the evolution of the relative phase as shown in Fig 3.b. By combining the evolution of the sideband power with the evolution of the relative phase, we can establish the phase portrait of the sideband, as displayed in Fig 3.c. Good agreement between experimental results (solid lines) and numerical simulations (dashed lines) is obtained, which validates the consistency of our measurement.
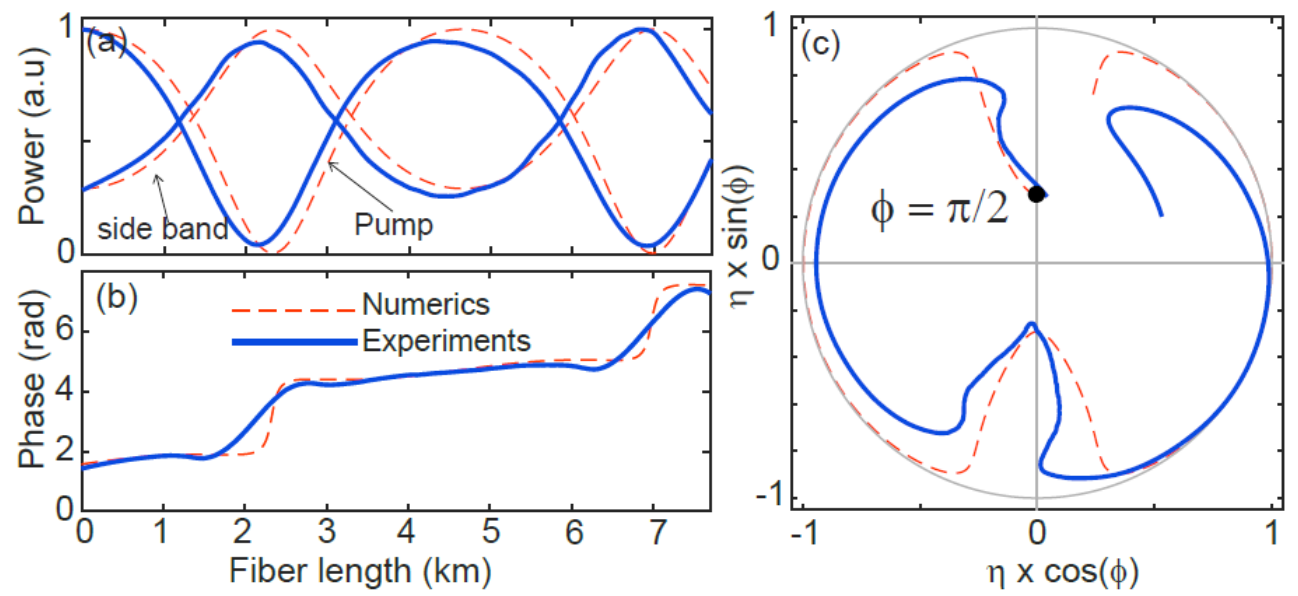

Fig. 3. Longitudinal evolution of (a) the normalized pump and sideband powers and of (b) the pump-sideband relative phase. (c) Phase portrait of the sideband corresponding to (a) and (b). Solid lines corresponds to experimental data and dashed one to numerics. Parameters: fibre length $\mathrm{L}=7.7 \mathrm{~km}, \beta_{2}=-19 \times 10^{-27} \mathrm{~s}^{2} / \mathrm{m}, \gamma=1.3 \times 10^{-3} / \mathrm{W} / \mathrm{m}, P_{p}=450 \mathrm{~mW}$, sideband to pump ratio $\eta=0.14$, sideband detuning $35 \mathrm{GHz}$. Powers are normalized to their maximum values.

In summary, our original novel experimental setup allowed us to perform the first complete distributed characterization, in phase and intensity, of the Fermi Pasta Ulam recurrence. We have been able to record the longitudinal evolution of the pump and sidebands intensities and their relative phase. Our setup is then a good test bed compare to other experimental systems to further investigate the nonlinear dynamics of modulation instability, that is directly with other complex nonlinear processes of high interest such as rogue waves, supercontinuum generation or frequency comb generation. 


\section{REFERENCES}

[1] V. E. Zakharov and L. A. Ostrovsky, “Modulation instability: The beginning”, Physica D 238, $540\{548$ (2009).

[2] N. Akhmediev, “Nonlinear physics - Deja vu in optics”, Nature 413, 267-268 (2001).

[3] G. Van Simaeys, P. Emplit, and M. Haelterman, "Experimental Demonstration of the Fermi-Pasta-Ulam Recurrence in a Modulationally Unstable Optical Wave," Phys. Rev. Lett. 87, 033902 (2001).

[4] A. Mussot, A. Kudlinski, M. Droques, P. Szriftgiser, and N. Akhmediev, "Fermi-Pasta-Ulam recurrence in nonlinear fibre optics: the role of reversible and irreversible losses”, Phys. Rev. X 4, 011054 (2014).

[5] S. Wabnitz and N. Akhmediev; "Efficient modulation frequency doubling by induced modulation instability", Opt. Comm. 283 (2010) 1152- 1154 